\title{
A highly-efficient oxygen evolution electrode based on defective nickel-iron layered double hydroxide
}

\author{
Xuya Xiong ${ }^{1}$, Zhao Cai ${ }^{1,2^{*}}$, Daojin Zhou ${ }^{1}$, Guoxin Zhang $^{3}$, Qian Zhang ${ }^{1}$, Yin Jia ${ }^{1,4}$, Xinxuan Duan ${ }^{1}$, \\ Qixian Xie ${ }^{1}$, Shibin Lai ${ }^{1}$, Tianhui Xie ${ }^{1}$, Yaping $\mathrm{Li}^{{ }^{1}}$, Xiaoming Sun ${ }^{1,4^{*}}$ and Xue Duan ${ }^{1}$
}

\begin{abstract}
Exploring efficient and cost-effective electrocatalysts for oxygen evolution reaction (OER) is critical to water splitting. While nickel-iron layered double hydroxide (NiFe LDH) has been long recognized as a promising nonprecious electrocatalyst for OER, its intrinsic activity needs further improvement. Herein, we design a highly-efficient oxygen evolution electrode based on defective NiFe LDH nanoarray. By combing the merits of the modulated electronic structure, more exposed active sites, and the conductive electrode, the defective NiFe LDH electrocatalysts show a low onset potential of $1.40 \mathrm{~V}$ ( $v s$. RHE). An overpotential of only $200 \mathrm{mV}$ is required for $10 \mathrm{~mA} \mathrm{~cm}^{-2}$, which is $48 \mathrm{mV}$ lower than that of pristine NiFe-LDH. Density functional theory plus $U$ $(D F T+U)$ calculations are further employed for the origin of this OER activity enhancement. We find the introduction of oxygen vacancies leads to a lower valance state of Fe and the narrowed bandgap, which means the electrons tend to be easily excited into the conduction band, resulting in the lowered reaction overpotential and enhanced OER performance.
\end{abstract}

Keywords: oxygen evolution reaction, layered double hydroxide, oxygen vacancy, electrocatalysis

\section{INTRODUCTION}

Hydrogen from water-splitting presents a clean and sustainable energy to replace traditional fossil energy and address the energy crisis as well as the environmental issues [1-3]. However, the low efficiency and sluggish kinetics of oxygen evolution reaction (OER) catalysts have restricted the large-scale production of hydrogen [4,5]. Precious metal catalysts (such as $\mathrm{IrO}_{2}$ and $\mathrm{RuO}_{2}$ ) have to be involved in the electrode [6-9] to lower the overpotential and facilitate the OER process, but the limited performance (e.g., an onset potential of $1.5 \mathrm{~V}$ ) has still hindered their commercialization [10-13]. Instead, 3d-transition metals ( $\mathrm{Ni}, \mathrm{Co}, \mathrm{Fe}$, etc.) have received increasing research interest owing to the earth abundance and considerable activity [14-19]. Notably, NiFe-based catalyst has shown the highest activity among the $3 \mathrm{~d}$ transition metal systems in alkaline conditions. Electrode design based on $\mathrm{NiFe}$ catalyst with further depressed onset potential and high energy efficiency is still very challenging [20-25].

In recent decades, layered double hydroxide $(\mathrm{LDH})$, highly tunable layered materials with brucite-like layers and intercalated anions, have been widely considered as promising OER electrode material [26-28]. Extensive efforts have been made to improve the OER activity of $\mathrm{NiFe}$ LDHs by tuning their chemical composition/nanostructures or enhancing the electrical conductivity [2932]. For example, $\mathrm{Hu}$ and co-workers [33] found an OER onset potential of $1.48 \mathrm{~V}$ for $\mathrm{NiFe} \mathrm{LDH}$ by exfoliating $\mathrm{LDH}$ to single-layer nanosheet and exposing more metal sites. Dai's group [34] has anchored NiFe LDHs on conductive carbon nanotube to enhance the conductivity, thus improving their OER activity with an early onset potential of $1.45 \mathrm{~V}$. Luo et al. [35] intercalated phosphorus oxoanions into $\mathrm{NiFe} \mathrm{LDH}$ to tune the surface electronic structure, and $1.45 \mathrm{~V}$ was also obtained for the onset potential. Moreover, by combining the abovementioned methods, the OER onset potential of $\mathrm{NiFe}$ $\mathrm{LDH}$ can be much improved, but still larger than $1.40 \mathrm{~V}$ so far [36]. On the other hand, exfoliation of $\mathrm{NiFe} \mathrm{LDH} /$ engineering the nanostructure commonly involved var-

\footnotetext{
${ }^{1}$ State Key Laboratory of Chemical Resource Engineering, Beijing University of Chemical Technology, Beijing 100029, China

${ }^{2}$ Department of Chemistry and Energy Sciences Institute, Yale University, 810 West Campus Drive, West Haven CT 06516, USA

${ }^{3}$ College of Electrical Engineering and Automation, Shandong University of Science and Technology, Tsingtao 266590, China

${ }^{4}$ College of Energy, Beijing Advanced Innovation Center for Soft Matter Science and Engineering, Beijing University of Chemical Technology, Beijing 100029, China

* Corresponding authors (emails: sunxm@mail.buct.edu.cn (Sun X); liyp@mail.buct.edu.cn (Li Y); caizki@126.com (Cai Z))
} 
ious organic solvents or harsh conditions like high temperature/pressure. Therefore, developing $\mathrm{NiFe} \mathrm{LDH}$ based electrode with higher intrinsic activity (especially the onset potential under $1.40 \mathrm{~V}$ ) in a mild and economical way is pretty urgent.

In this work, we report a highly-efficient OER electrode based on defective NiFe LDHs. After a simple immersion in $\mathrm{NaBH}_{4}$ solution at room temperature, oxygen vacancies were introduced to NiFe $\mathrm{LDH}$ nanoarray. More importantly, the as-designed defective $\mathrm{NiFe} \mathrm{LDH}$ electrode exhibited a significantly improved OER activity with an early onset potential of $1.40 \mathrm{~V}$ and a small overpotential of $200 \mathrm{mV}$ for $10 \mathrm{~mA} \mathrm{~cm}$. The defective $\mathrm{NiFe}$ LDH was prepared under a mild reduction condition but surprisingly stable even after $10 \mathrm{~h}$ continued OER catalysis. Based on X-ray photoelectron spectroscopy (XPS)/electrochemical analysis and $\mathrm{DFT}+U$ calculations, the excellent OER performance of defective $\mathrm{NiFe}$ LDH electrode should be attributed to the tuned electronic structure and more exposed active sites. Our study not only demonstrates defective NiFe LDH nanoarray as highly-active and cost-effective OER electrode, but also shed light on fabricating highly-efficient electrode for large-scale industrial applications in an economic way.

\section{EXPERIMENTAL SECTION}

\section{Chemicals and characterizations}

$\mathrm{Ni}\left(\mathrm{NO}_{3}\right)_{2} \cdot 6 \mathrm{H}_{2} \mathrm{O}$ and $\mathrm{Fe}\left(\mathrm{NO}_{3}\right)_{3} \cdot 9 \mathrm{H}_{2} \mathrm{O}$ were purchased from Sinopharm Chemical Reagent Co, Ltd. (SCRC). $\mathrm{CO}\left(\mathrm{NH}_{2}\right)_{2}$ were purchased from Beijing Chemical Reagents Company. $\mathrm{NaBH}_{4}$ was purchased from Tianjin East China Reagent Factory. Nickel foam was purchased from Shanxi Li Zhiyuan Battery Materials Ltd. Deionized water with a resistivity $>18 \mathrm{M} \Omega$ was used to prepare all aqueous solutions. All the reagents were of analytical grade and were used without further purification.

The morphologies of as-prepared samples were characterized by scanning electron microscope (SEM; Zeiss SUPRA 55) and high-resolution transmission electron microscope (HRTEM; JEOL JEM-2100). X-ray diffraction (XRD) patterns were collected on Rigaku X-ray diffractometer (UItima III, $3 \mathrm{~kW}$ ), recorded with $2 \theta$ ranging from $3^{\circ}$ to $80^{\circ}$. XPS measurements were carried out with a PHI Quantera II XPS Scanning Microprobe. Electron Paramagnetic Resonance (EPR) was conducted on ELEXSYS-II, Bruker.

\section{Synthesis of defective NiFe LDH nanoarray}

The NiFe LDH nanoarray was prepared by hydrothermal method. In a typical synthesis, $0.33 \mathrm{mmol} \mathrm{Fe}\left(\mathrm{NO}_{3}\right)_{3} \cdot 9 \mathrm{H}_{2} \mathrm{O}$, $0.66 \mathrm{mmol} \mathrm{Ni}\left(\mathrm{NO}_{3}\right)_{2} \cdot 6 \mathrm{H}_{2} \mathrm{O}$ and $10 \mathrm{mmol} \mathrm{CO}\left(\mathrm{NH}_{2}\right)_{2}$ were dissolved in $35 \mathrm{~mL}$ distilled water and stirred to form a clear solution. Nickel foam (about $3 \mathrm{~cm} \times 2 \mathrm{~cm}$ ) was carefully cleaned with concentrated $\mathrm{HCl}$ solution (37 wt $\%$ ) in an ultrasound bath for 5 min with the purpose of removing the surface nickel oxide layer, and then washed by deionized water and ethanol respectively for $5 \mathrm{~min}$ to remove the remnant acid. The solution and the nickel foam were transferred to a $40 \mathrm{~mL}$ Teflon-lined stainlesssteel autoclave, which was sealed, maintained at $120^{\circ} \mathrm{C}$ for $12 \mathrm{~h}$, and then allowed to cool to room temperature naturally. A brown NiFe LDH nanoarray was formed and subsequently rinsed with distilled water, ethanol for 5 min ultrasonically. The prepared $\mathrm{NiFe} \mathrm{LDH}$ array was dried in the air. To prepare defective $\mathrm{NiFe} \mathrm{LDH}$ nanoarray, $8 \mathrm{mmol} \mathrm{NaBH}_{4}$ was added into $40 \mathrm{~mL}$ distilled water and slightly stirred to form a clear solution. The $\mathrm{NiFe} \mathrm{LDH}$ array was cut into a rectangle shape (about $1 \mathrm{~cm} \times 2 \mathrm{~cm})$ and immersed in the $0.2 \mathrm{~mol} \mathrm{~L}^{-1} \mathrm{NaBH}_{4}$ solution for a certain time (5-60 $\mathrm{min})$ at room temperature. Afterwards, the reduced sample was rinsed with distilled water, ethanol for 5 min ultrasonically and then dried for further use.

\section{Electrochemical measurements}

The electrochemical measurements were performed at room temperature in a three-electrode system in $1 \mathrm{~mol} \mathrm{~L}^{-1}$ aqueous $\mathrm{KOH}$ solution using an electrochemical workstation (CHI 660E, Chenhua, Shanghai). The defective NiFe LDH nanoarray on the Nickle foam $(1 \mathrm{~cm} \times 1 \mathrm{~cm})$ was used as a working electrode. $\mathrm{A} \mathrm{Hg} /$ $\mathrm{HgO}$ electrode and a platinum plate were used as the reference and the counter electrode, respectively. The electrolyte was saturated by oxygen bubbles (for OER) before and during the experiments. The polarization data were collected using linear sweep voltammetry at a scan rate of $1 \mathrm{mV} \mathrm{s}^{-1}$ after twenty cyclic voltammetric scans. The electrochemical impedance spectroscopy was obtained by AC impedance spectroscopy in $1 \mathrm{~mol} \mathrm{~L}^{-1} \mathrm{KOH}$ solution at open circuit voltage from $10^{5}-0.1 \mathrm{~Hz}$ with an AC voltage of $5 \mathrm{mV}$. The stability of the electrode was measured by applying a constant potential of $1.43 \mathrm{~V}$ for $10 \mathrm{~h}$.

\section{Calculation methods}

$\mathrm{DFT}+U$ calculations were employed to analyze the role of oxygen vacancies in $\mathrm{NiFe} \mathrm{LDH}$ structure during the OER progress. The (100) surface was take into consideration as the surface corresponds to the edge of LDH structure 
mostly. For pristine NiFe-LDH (100) facet, Fe atom was used as the active site for OER. To simulate the oxygen vacancy, an $-\mathrm{OH}$ group was removed from the top layer of (100), the consequently exposed Fe-bridge site was used as the active site for OER.

All calculations were performed using the projectoraugmented wave method and a plane-wave basis set as implemented in the Vienna Ab Initio Simulation Package (VASP). The bulk and surface properties of NiFe LDH were optimized within GGA-PBE. A full optimization of all atom positions in the bulk was performed via the action of a conjugate gradient optimization procedure. The bulk lattice constants were optimized using the $3 \times 3 \times 1$ Monkhorst-Pack $k$-point sampling. The cutoff energy for plane-wave basis functions was set to $400 \mathrm{eV}$ with the energy change convergence criterion of $1 \times 10^{-4} \mathrm{eV}$. Atomic positions were allowed to relax until the sum of the absolute forces is less than $0.05 \mathrm{eV} \AA^{-1}$. Hubbard- $U$ correction method was applied to improve the description of localized $\mathrm{Ni}$ and $\mathrm{Fe}$ d-electrons in the NiFe-LDH with $U=5.3$ and $U=6.45$ for $\mathrm{Fe}$ and $\mathrm{Ni}$, respectively. Spin polarization was also considered in all the calculations.

\section{RESULTS AND DISSCUSSION}

Fig. 1 illustrated the fabrication process of defective $\mathrm{NiFe}$ LDH nanoarray. The pristine NiFe LDH (here denoted as "P-NiFe LDH") was mildly reduced by $0.2 \mathrm{~mol} \mathrm{~L}^{-1} \mathrm{NaBH}_{4}$ solution at room temperature to produce reduced $\mathrm{NiFe}$ $\mathrm{LDH}$ (here denoted as "R-NiFe LDH"). The color of P$\mathrm{NiFe}$ LDH nanoarray (brown) changed to yellow-grey after a reduction for $30 \mathrm{~min}$ and even changed into grey $60 \mathrm{~min}$ later (Fig. S1). Such color change implied a tuned chemical/electronic structure of P-NiFe LDH.

SEM characterization was carried out to look into the morphology change of NiFe LDH during the reduction. As shown in Fig. 2a, the P-NiFe LDHs were sheet-like and showed an oriented 3D array structure. Such open 3D structure means adequate exposure of edge sites and efficient use of the active materials for catalysis. After the immersion in $\mathrm{NaBH}_{4}$ solution for $10 \mathrm{~min}$, although nanosheet morphology and 3D array structure were roughly preserved, the surface of R-NiFe LDH turned to be coarse and nanoporous (Fig. 2b). This suggested a surface reaction during the reduction, which led to a slightly destroyed crystal structure, implying a defective nature. The HRTEM images and electron diffractions (ED) of P- and $\mathrm{R}-\mathrm{NiFe} \mathrm{LDH}$ also confirmed this point, in the inset in Fig. 2a, b and Fig. S2, discontinuous lattices and weakened ED patterns could be clearly seen after the mild reduction.

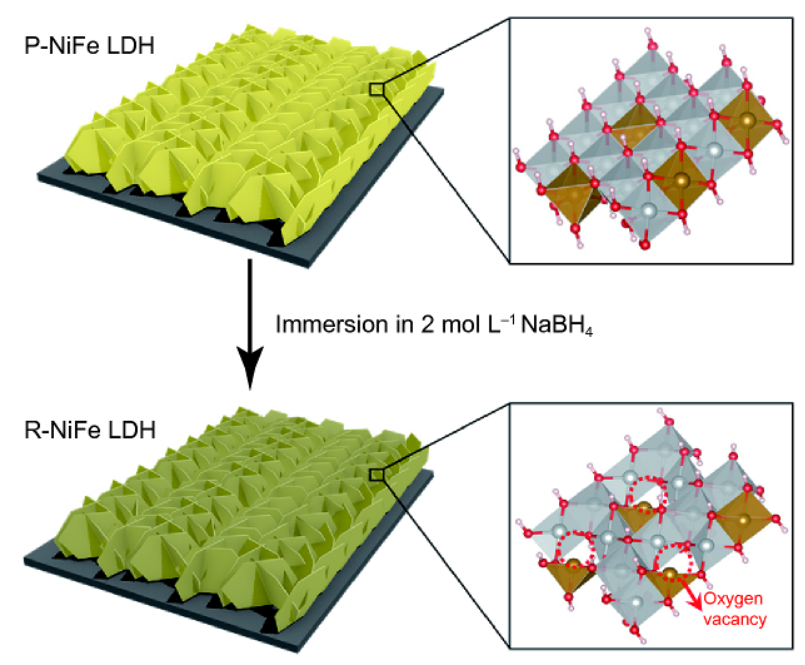

Figure 1 Schematic illustration of introducing oxygen vacancy defects to $\mathrm{NiFe}$ LDH nanoarray electrode.

Further extending the reduction time to $60 \mathrm{~min}$, the R$\mathrm{NiFe} \mathrm{LDH}$ nanosheets were collapsed into irregular nanoparticles (Fig. S3), demonstrating a gradually spoiled structure. XRD analysis (Fig. $2 c$ and Fig. S4a) showed the diffraction peaks of P-NiFe LDH matched well with the pure hexagonal-phased NiFe LDH (black lines, PDF\#400215), meanwhile, the R-NiFe LDH exhibited a weaker but the same diffraction pattern as $\mathrm{P}-\mathrm{NiFe} \mathrm{LDH}$, suggesting no phase destruction happened during the $10 \mathrm{~min}$ mild reduction process. However, the R-NiFe LDH diffraction peaks turned relatively broad, suggesting the wider distribution of their lattice spacing and a disordered LDH structure with defects, consistent with our HRTEM and ED results. Energy Dispersive Spectrometer (EDS) analysis (Fig. S4b, c) showed the $\mathrm{Ni} / \mathrm{Fe}$ ratio in $\mathrm{R}$ $\mathrm{NiFe} \mathrm{LDH}$ is 2.98, quite similar to that of P-NiFe LDH (2.96). Besides, no boron spices were detected in R-NiFe $\mathrm{LDH}$, suggesting no metal boride formation or dopedboron in the R-NiFe LDH structure.

First derivative X-band EPR spectra (Fig. 2d) was carried out to confirm the defect structure of R-NiFe LDH. While the EPR signal of P-NiFe LDH was relatively weak (due to the very limited defects), a broad and strong signal of $\mathrm{R}-\mathrm{NiFe} \mathrm{LDH}$ was detected, demonstrating a defect-rich structure [37]. Moreover, the signals of R$\mathrm{NiFe} \mathrm{LDH}$ at $g=1.99$ could be identified as the electrons trapped on oxygen vacancies, indicating the defects in $\mathrm{R}$ $\mathrm{NiFe}$ LDH were oxygen vacancies [38]. Further evidence came from the XPS characterizations. In the $\mathrm{O}$ 1s spectrum (Fig. 2e), the peak at $531.1 \mathrm{eV}$ is attributed to the unsaturated oxygen species [39], suggesting R-NiFe LDHs 

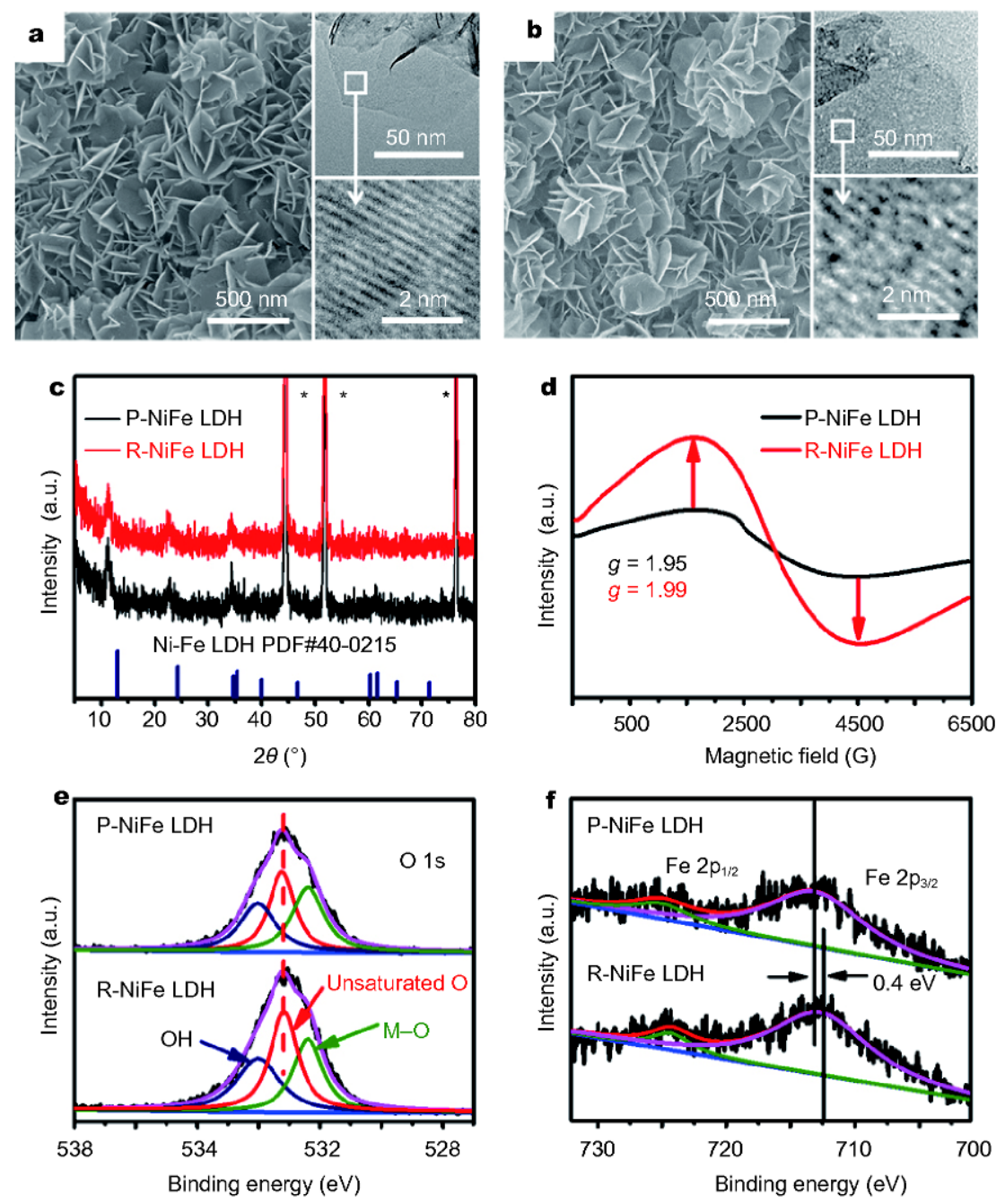

Figure 2 SEM images of as-prepared (a) P-NiFe LDH and (b) R-NiFe LDH. The insets are TEM and HRTEM images of P- and R-NiFe LDH, implying the structure with defects after reduction. (c) XRD patterns and (d) EPR characterization of P-NiFe LDH and R-NiFe LDH. The peak marked "*” presented Ni foam. (e) O 1s and (f) Fe 2p XPS spectra of of P- and R-NiFe LDH, the peak at $531.1 \mathrm{eV}$ was assigned to defective oxygen species.

were rich in oxygen vacancies, which was in accordance with our EPR findings [40]. The Fe and Ni 2p spectra were also examined to get better understanding of the RNiFe LDH electrode, as shown in Fig. $2 \mathrm{f}$ and Fig. S5. While peaks at $\sim 855.7 \mathrm{eV}$ and $\sim 713.3 \mathrm{eV}$ were attributed to $\mathrm{Ni}^{2+} 2 \mathrm{p}_{3 / 2}$ and $\mathrm{Fe}^{3+} 2 \mathrm{p}_{3 / 2}$, respectively, there was a small peak shift for both $\mathrm{Ni}(0.5 \mathrm{eV})$ and $\mathrm{Fe}(0.4 \mathrm{eV})$ toward lower binding energies in $\mathrm{R}-\mathrm{NiFe} \mathrm{LDH}$, suggesting a decrease in the oxidation state of the transition metals, indicating that the introduction of oxygen vacancies were balanced by the lowered valence state of $\mathrm{Ni}$ and $\mathrm{Fe}$ sites.

The electrocatalytic OER activity of the as-prepared RNiFe LDH electrode was investigated in alkaline solutions $\left(1 \mathrm{~mol} \mathrm{~L}^{-1} \mathrm{KOH}\right)$ using a standard three-electrode system.
IR-corrected OER polarization curves were recorded at a slow scan rate of $1 \mathrm{mV} \mathrm{s}^{-1}$ to minimize the capacitive current in order to compare the intrinsic activity of the RNiFe LDHs. As shown in Fig. 3a, b, R-NiFe LDHs outperformed the P-NiFe LDH counterpart in terms of onset potential and Tafel slope. It should be noted that with a reduction time of 10 min (i.e., R10), the R10-NiFe LDH sample exhibited the best OER performance with an early onset potential of $1.40 \mathrm{~V}$ and a small overpotential of 200 $\mathrm{mV}$ for $10 \mathrm{~mA} \mathrm{~cm}{ }^{-2}$, which is the best NiFe LDH electrocatalyst to date (Table S1). The OER Faraday efficiency was determined to be $99.4 \%$, further demonstrating the high intrinsic activity of R-NiFe LDH 10 min. However, when the R-NiFe LDHs were over-reduced (more than 

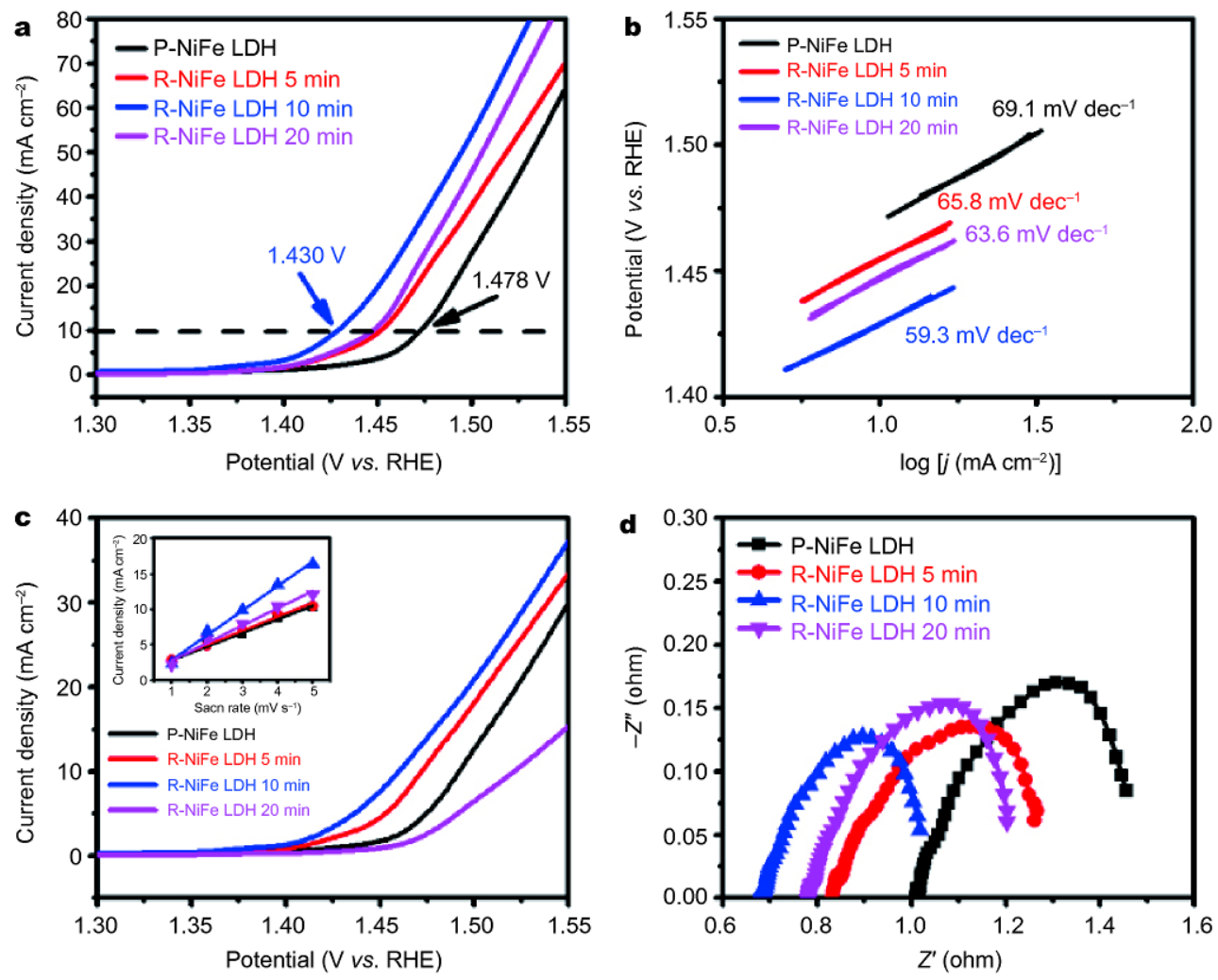

Figure 3 (a) Linear sweep voltammetry polarization curves of as-prepared P- and R-NiFe LDHs, the insets shows the overpotential at $10 \mathrm{~mA} \mathrm{~cm}^{-2}$ for $\mathrm{NiFe}$ LDH with different reduction times, the error bar was obtained by repeating the test for at least five times. (b) Tafel plots of as-prepared P- and R-NiFe LDHs. (c) Linear sweep voltammetry polarization curves of as-prepared P- and R-NiFe LDHs normalized by their ECSA. The insets shows the $C_{\mathrm{dl}}$ calculations of the P- and R-NiFe LDHs. (d) Nyquist plots of the P- and R-NiFe LDHs.

$10 \mathrm{~min}$ ), the OER performance turned worse than that of R10-NiFe LDH (Figs S6 and S7). This should be attributed to the partly destroyed structure of $\mathrm{LDH}$, as demonstrated by SEM and XRD results. The electrochemical double layer capacitance $\left(C_{\mathrm{dl}}\right)$ was monitored to trace the source for such OER activity enhancement, as this parameter fully reflects the electrochemical surface active area (ECSA). Figs S7, S8, Table S2 and the inset of Fig. 3c showed that R10 -NiFe LDH possessed the highest $C_{\mathrm{dl}}$ among the reduced catalysts, suggesting the enhanced OER performance of R$\mathrm{NiFe} \mathrm{LDH}$ was partly due to the enlarged ECSA induced by oxygen defects. After normalizing the OER current density by ECSA (Fig. 3c), the R-NiFe LDHs showed very similar activity (Fig. 3a), suggesting the higher intrinsic activity of the R-NiFe LDHs. Furthermore, electrochemical impedance spectroscopy (EIS) was also performed to study the electrode kinetics of such R-NiFe LDH electrode in OER. The R10-NiFe LDH electrode showed a smaller charge transfer resistance $(\sim 0.36 \Omega)$ than that of P-NiFe LDH $(\sim 0.52 \Omega)$, demonstrating an accelerated charge transfer process for $\mathrm{R} 10-\mathrm{NiFe} \mathrm{LDH}$ electrode, which should be beneficial for the improved OER performance. Besides, the solution resistance of R10-NiFe LDH electrode $(\sim 0.69 \Omega)$ was smaller than that of P-NiFe LDH $(\sim 1.05 \Omega)$, suggesting higher electrical conductivity of R-NiFe $\mathrm{LDH}$, leading to the reduction of the overpotential. This electrochemical analysis confirmed the outstanding OER performance of as-prepared $\mathrm{R}-\mathrm{NiFe} \mathrm{LDH}$ electrode and suggested such high intrinsic activity enhancement is attributed to the introduced oxygen vacancies, which led to a higher intrinsic activity, larger ECSA, as well as better electro-conductivity.

$\mathrm{DFT}+U$ calculations are utilized to understand the thermodynamic/electronic details of the OER process on the (100) surface of the R-NiFe LDH and to get further insight into the oxygen vacancy effect on the OER performance of R-NiFe LDHs since (100) has been widely accepted as the most active facets in LDH structure [41]. We applied a standard four electrons OER mechanism proposed by J. K. Nørskov and A. T. Bell [42], in which $\mathrm{H}_{2} \mathrm{O}$ is chosen as the OER reactant. $-\mathrm{OH}$ groups on the 

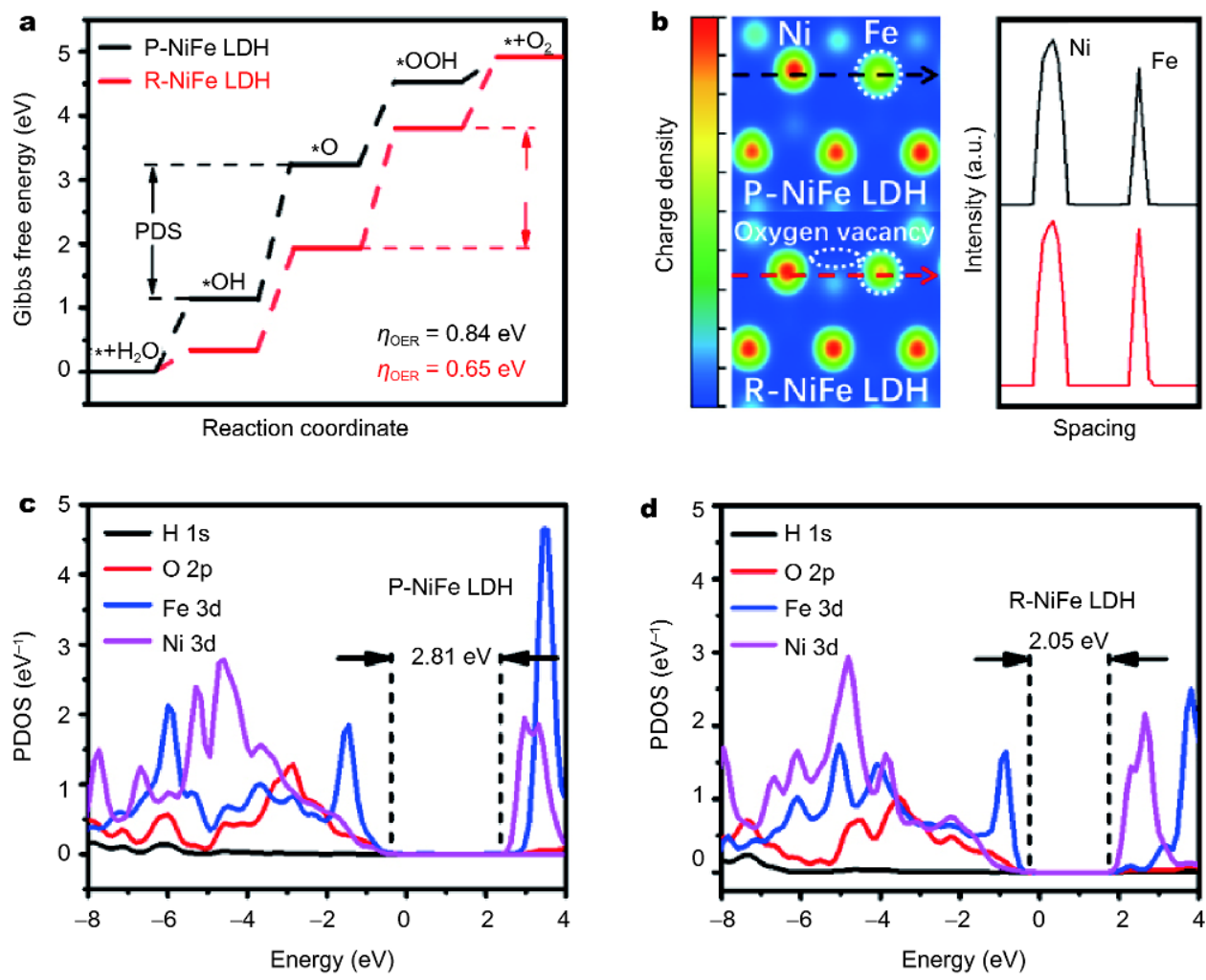

Figure 4 Calculated results of OER process on P-NiFe LDH and R-NiFe LDH structure. (a) Free energy plots and (b) charge density analysis on PNiFe LDH and R-NiFe LDH for OER. Partial density of state results of (c) P-NiFe LDH, and (d) R-NiFe LDH.

(100) surface of pristine NiFe LDH structure were removed to simulate the oxygen vacancies. Consequently, the bridge site between $\mathrm{Ni}$ and $\mathrm{Fe}$ was proposed to be the active site for OER (Fig. S11) [43]. The OER on NiFe catalysts' surface involved a four-electron process, as revealed in Fig. 4a, and the potential determining step (PDS) of pristine NiFe LDH was found to be the deprotonation step with a high overpotential of $0.86 \mathrm{~V}$. However, with oxygen vacancy introduced, the PDS of R-NiFe $\mathrm{LDH}$ turned to be the $\mathrm{OOH}$ formation step with an overpotential of $0.65 \mathrm{~V}$, which is much lower than that of pristine $\mathrm{NiFe} \mathrm{LDH}$, demonstrating the higher intrinsic activity. Moreover, partial density of states (PDOS) and Bader charge analysis are carried out to study the electronic state of NiFe $\mathrm{LDH}$ structure with oxygen vacancies $[44,45]$. We further applied the Bader charge analysis to investigate the electronic structure of R-NiFe $\mathrm{LDH}$, as shown in Fig. 4b. After the introduction of oxygen vacancies, the electronic density of Fe increased, demonstrating a lowered valence state, which was in accordance with our XPS results. Meanwhile, in the valance band of P-NiFe LDH (Fig 4c), the peak at $-6 \mathrm{eV}$ indicated that Fe $3 \mathrm{~d}$ was in a low spin high valance state. Compared with that of R-NiFe LDH (Fig. 4d), the peak of Fe 3d shifted to $-5 \mathrm{eV}$, indicating a high spin low valance state after the introduction of oxygen vacancy. Such low valance states of $\mathrm{Fe}$ in $\mathrm{R}-\mathrm{NiFe} \mathrm{LDH}$ are consistent with our experimental findings (XRS in Fig. 2d). Besides, the band gap energy of R-NiFe LDH structure turned out to be $2.05 \mathrm{eV}$, which was smaller than that of P-NiFe LDH $(2.81 \mathrm{eV})$, suggesting a better electro-conductivity of R-NiFe LDH $[46,47]$. Thus, our theory and experiment came to a good agreement, suggesting that by introducing oxygen vacancy defects in NiFe LDH electrode, the electronic structures on the catalysts' surface were finely tuned and the electrical conductivity was improved, finally leading to the significantly enhanced OER activity.

\section{CONCLUSIONS}

In summary, we introduced oxygen vacancies to $\mathrm{NiFe}$ LDH nanoarray by $\mathrm{NaBH}_{4}$ at room temperature in aqueous solution, thus developing a highly-efficient electrode for OER. XPS/electrochemical analysis, together with $D F T+U$ calculations, suggested the modulated electronic structure, more exposed active sites, and the improved electrical conductivity for $\mathrm{R}-\mathrm{NiFe} \mathrm{LDH}$ are responsible 
for the significantly enhanced, almost the best, $\mathrm{NiFe}$ LDH-based OER activity. This study demonstrates that the defective NiFe LDH nanoarray as highly-active and cost-effective OER electrode plays an important role in boosting electrocatalytic activities, thus shedding light on advanced electrode design towards large-scale hydrogen production via electrochemical water splitting.

\section{Received 22 November 2017; accepted 13 January 2018; published online 11 February 2018}

1 Luo J, Im JH, Mayer MT, et al. Water photolysis at $12.3 \%$ efficiency via perovskite photovoltaics and Earth-abundant catalysts. Science, 2014, 345: 1593-1596

2 Walter MG, Warren EL, McKone JR, et al. Solar water splitting cells. Chem Rev, 2010, 110: 6446-6473

3 Khan SUM, Al-Shahry M, Ingler WB. Efficient photochemical water splitting by a chemically modified $\mathrm{n}-\mathrm{TiO}_{2}$. Science, 2002, 297: 2243-2245

4 Kanan MW, Nocera DG. In situ formation of an oxygen-evolving catalyst in neutral water containing phosphate and $\mathrm{Co}^{2+}$. Science, 2008, 321: 1072-1075

5 McCrory CCL, Jung S, Peters JC, et al. Benchmarking heterogeneous electrocatalysts for the oxygen evolution reaction. J Am Chem Soc, 2013, 135: 16977-16987

6 Fang YH, Liu ZP. Mechanism and tafel lines of electro-oxidation of water to oxygen on $\mathrm{RuO}_{2}$ (110). J Am Chem Soc, 2010, 132: 1821418222

7 Lee Y, Suntivich J, May KJ, et al. Synthesis and activities of rutile $\mathrm{IrO}_{2}$ and $\mathrm{RuO}_{2}$ nanoparticles for oxygen evolution in acid and alkaline solutions. J Phys Chem Lett, 2012, 3: 399-404

8 Reier T, Oezaslan M, Strasser P. Electrocatalytic oxygen evolution reaction (OER) on $\mathrm{Ru}, \mathrm{Ir}$, and Pt catalysts: A comparative study of nanoparticles and bulk materials. ACS Catal, 2012, 2: 1765-1772

9 Lettenmeier P, Wang L, Golla-Schindler U, et al. Nanosized $\mathrm{IrO}_{x}$-Ir catalyst with relevant activity for anodes of proton exchange membrane electrolysis produced by a cost-effective procedure. Angew Chem Int Ed, 2016, 55: 742-746

10 Zhao Y, Jia X, Waterhouse GIN, et al. Layered double hydroxide nanostructured photocatalysts for renewable energy production. Adv Energy Mater, 2016, 6: 1501974

11 Cao R, Lee JS, Liu M, et al. Recent progress in non-precious catalysts for metal-air batteries. Adv Energy Mater, 2012, 2: 816-829

12 Prabu M, Ketpang K, Shanmugam S. Hierarchical nanostructured $\mathrm{NiCO}_{2} \mathrm{O}_{4}$ as an efficient bifunctional non-precious metal catalyst for rechargeable zinc-air batteries. Nanoscale, 2014, 6: 3173-3181

13 Cui X, Ren P, Deng D, et al. Single layer graphene encapsulating non-precious metals as high-performance electrocatalysts for water oxidation. Energy Environ Sci, 2016, 9: 123-129

14 Liang H, Meng F, Cabán-Acevedo M, et al. Hydrothermal continuous flow synthesis and exfoliation of NiCo layered double hydroxide nanosheets for enhanced oxygen evolution catalysis. Nano Lett, 2015, 15: 1421-1427

15 Kargar A, Yavuz S, Kim TK, et al. Solution-processed $\mathrm{CoFe}_{2} \mathrm{O}_{4}$ nanoparticles on $3 \mathrm{D}$ carbon fiber papers for durable oxygen evolution reaction. ACS Appl Mater Interfaces, 2015, 7: 17851-17856

16 Chen Z, Zhao $\mathrm{H}$, Zhang J, et al. IrNi nanoparticle-decorated flower-shaped $\mathrm{NiCO}_{2} \mathrm{O}_{4}$ nanostructures: controllable synthesis and enhanced electrochemical activity for oxygen evolution reaction. Sci China Mater, 2017, 60: 119-130

17 Zhang $\mathrm{F}$, Shi $\mathrm{Y}$, Xue $\mathrm{T}$, et al. In situ electrochemically converting $\mathrm{Fe}_{2} \mathrm{O}_{3}-\mathrm{Ni}(\mathrm{OH})_{2}$ to $\mathrm{NiFe}_{2} \mathrm{O}_{4}$-NiOOH: a highly efficient electrocatalyst towards water oxidation. Sci China Mater, 2017, 60: 324334

18 Guo S, Yang Y, Liu N, et al. One-step synthesis of cobalt, nitrogencodoped carbon as nonprecious bifunctional electrocatalyst for oxygen reduction and evolution reactions. Sci Bull, 2016, 61: 68-77

19 Zhao Y, Jia X, Chen G, et al. Ultrafine $\mathrm{NiO}$ nanosheets stabilized by $\mathrm{TiO}_{2}$ from monolayer NiTi-LDH precursors: An active water oxidation electrocatalyst. J Am Chem Soc, 2016, 138: 6517-6524

20 Louie MW, Bell AT. An investigation of thin-film Ni-Fe oxide catalysts for the electrochemical evolution of oxygen. J Am Chem Soc, 2013, 135: 12329-12337

21 Görlin M, Chernev P, Ferreira de Araújo J, et al. Oxygen evolution reaction dynamics, faradaic charge efficiency, and the active metal redox states of Ni-Fe oxide water splitting electrocatalysts. J Am Chem Soc, 2016, 138: 5603-5614

22 Li P, Xie Q, Zheng L, et al. Topotactic reduction of layered double hydroxides for atomically thick two-dimensional non-noble-metal alloy. Nano Res, 2017, 10: 2988-2997

23 Wang Q, Shang L, Shi R, et al. NiFe layered double hydroxide nanoparticles on $\mathrm{Co}, \mathrm{N}$-codoped carbon nanoframes as efficient bifunctional catalysts for rechargeable zinc-air batteries. Adv Energy Mater, 2017, 7: 1700467

24 Liu R, Wang Y, Liu D, et al. Water-plasma-enabled exfoliation of ultrathin layered double hydroxide nanosheets with multivacancies for water oxidation. Adv Mater, 2017, 29: 1701546

25 Wang Y, Zhang Y, Liu Z, et al. Layered double hydroxide nanosheets with multiple vacancies obtained by dry exfoliation as highly efficient oxygen evolution electrocatalysts. Angew Chem Int Ed, 2017, 56: 5867-5871

26 Fan G, Li F, Evans DG, et al. Catalytic applications of layered double hydroxides: recent advances and perspectives. Chem Soc Rev, 2014, 43: 7040-7066

27 Wang Q, O'Hare D. Recent advances in the synthesis and application of layered double hydroxide (LDH) nanosheets. Chem Rev, 2012, 112: 4124-4155

28 Liu Z, Ma R, Osada M, et al. Synthesis, anion exchange, and delamination of $\mathrm{Co}-\mathrm{Al}$ layered double hydroxide: assembly of the exfoliated nanosheet/polyanion composite films and magneto-optical studies. J Am Chem Soc, 2006, 128: 4872-4880

29 Ma W, Ma R, Wang C, et al. A superlattice of alternately stacked $\mathrm{Ni}-\mathrm{Fe}$ hydroxide nanosheets and graphene for efficient splitting of water. ACS Nano, 2015, 9: 1977-1984

30 Hunter BM, Hieringer W, Winkler JR, et al. Effect of interlayer anions on $[\mathrm{NiFe}]-\mathrm{LDH}$ nanosheet water oxidation activity. Energy Environ Sci, 2016, 9: 1734-1743

31 Wang Z, Zeng S, Liu W, et al. Coupling molecularly ultrathin sheets of NiFe-layered double hydroxide on $\mathrm{NiCo}_{2} \mathrm{O}_{4}$ nanowire arrays for highly efficient overall water-splitting activity. ACS Appl Mater Interfaces, 2017, 9: 1488-1495

32 Yu X, Zhang M, Yuan W, et al. A high-performance three-dimensional Ni-Fe layered double hydroxide/graphene electrode for water oxidation. J Mater Chem A, 2015, 3: 6921-6928

33 Song $\mathrm{F}, \mathrm{Hu} \mathrm{X}$. Exfoliation of layered double hydroxides for enhanced oxygen evolution catalysis. Nat Commun, 2014, 5: 4477

34 Gong $\mathrm{M}$, Li Y, Wang $\mathrm{H}$, et al. An advanced $\mathrm{Ni}-\mathrm{Fe}$ layered double hydroxide electrocatalyst for water oxidation. J Am Chem Soc, 
2013, 135: 8452-8455

35 Luo M, Cai Z, Wang C, et al. Phosphorus oxoanion-intercalated layered double hydroxides for high-performance oxygen evolution. Nano Res, 2017, 10: 1732-1739

36 Jia Y, Zhang L, Gao G, et al. A heterostructure coupling of exfoliated $\mathrm{Ni}-\mathrm{Fe}$ hydroxide nanosheet and defective graphene as a bifunctional electrocatalyst for overall water splitting. Adv Mater, 2017, 29: 1700017

37 Sun Y, Gao S, Lei F, et al. Atomically-thin two-dimensional sheets for understanding active sites in catalysis. Chem Soc Rev, 2015, 44: 623-636

38 Zuo F, Wang L, Wu T, et al. Self-doped $\mathrm{Ti}^{3+}$ enhanced photocatalyst for hydrogen production under visible light. J Am Chem Soc, 2010, 132: 11856-11857

39 Bao J, Zhang X, Fan B, et al. Ultrathin spinel-structured nanosheets rich in oxygen deficiencies for enhanced electrocatalytic water oxidation. Angew Chem Int Ed, 2015, 54: 7399-7404

$40 \mathrm{Xu} \mathrm{L}$, Jiang Q, Xiao Z, et al. Plasma-engraved $\mathrm{Co}_{3} \mathrm{O}_{4}$ nanosheets with oxygen vacancies and high surface area for the oxygen evolution reaction. Angew Chem Int Ed, 2016, 55: 5277-5281

41 Liao P, Keith JA, Carter EA. Water oxidation on pure and doped hematite (0001) surfaces: prediction of $\mathrm{Co}$ and $\mathrm{Ni}$ as effective dopants for electrocatalysis. J Am Chem Soc, 2012, 134: 1329613309

42 Friebel D, Louie MW, Bajdich M, et al. Identification of highly active $\mathrm{Fe}$ sites in $(\mathrm{Ni}, \mathrm{Fe}) \mathrm{OOH}$ for electrocatalytic water splitting. J Am Chem Soc, 2015, 137: 1305-1313

43 Chen J, Selloni A. First principles study of cobalt (hydr)oxides under electrochemical conditions. J Phys Chem C, 2013, 117: 20002-20006

44 Dong Y, Zhang P, Kou Y, et al. A first-principles study of oxygen formation over NiFe-layered double hydroxides surface. Catal Lett,
2015, 145: 1541-1548

45 Tao L, Lin CY, Dou S, et al. Creating coordinatively unsaturated metal sites in metal-organic-frameworks as efficient electrocatalysts for the oxygen evolution reaction: Insights into the active centers. Nano Energy, 2017, 41: 417-425

46 Xiao Z, Wang Y, Huang YC, et al. Filling the oxygen vacancies in $\mathrm{Co}_{3} \mathrm{O}_{4}$ with phosphorus: an ultra-efficient electrocatalyst for overall water splitting. Energy Environ Sci, 2017, 10: 2563-2569

47 Wang Y, Zhou T, Jiang $\mathrm{K}$, et al. Reduced mesoporous $\mathrm{Co}_{3} \mathrm{O}_{4}$ nanowires as efficient water oxidation electrocatalysts and supercapacitor electrodes. Adv Energy Mater, 2014, 4: 1400696

Acknowledgements This work was supported by the National Natural Science Foundation of China, National Key Research and Development Project (2016YFC0801302, 2016YFF0204402), the Program for Changjiang Scholars and Innovative Research Team in the University, and the Fundamental Research Funds for the Central Universities, and the longterm subsidy mechanism from the Ministry of Finance and the Ministry of Education of China.

Author contributions Sun X, Xiong X, and Cai Z conceived the project and designed the experiments. Xiong X, Zhou D, Jia Y, Lai S and Xie $\mathrm{Q}$ conducted material synthesis and electrochemical measurements. Xie $T$ performed SEM and TEM studies. Zhang Q performed XPS studies. Xiong X, Duan X, and Li Y carried out theoretical calculations. Sun X, Li Y, Cai Z, Duan X, and Zhang G co-wrote the paper. All authors discussed the results and commented on the manuscript.

Conflict of interest The authors declare no conflict of interest.

Supplementary information Supporting data are available in the online version of the paper. 

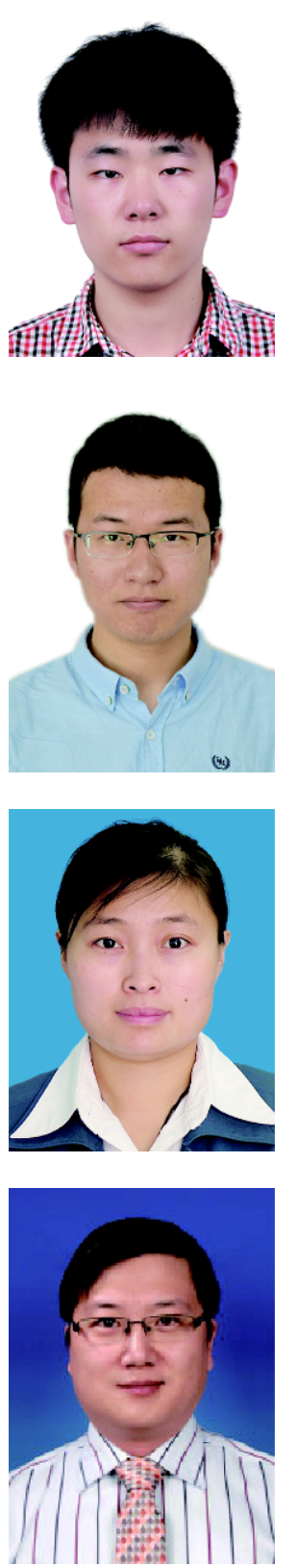

Xuya Xiong is currently a graduate student at the State Key Laboratory of Chemical Resource Engineering, Beijing University of Chemical Technology. His research interests focus on the transition metal oxide/hydroxide nanoarrays for electrocatalysis and DFT modeling studies.
Zhao Cai received his BSc degree from Beijing University of Chemical Technology in 2012, he is now a joint training PhD student at Beijing University of Chemical Technology in the group of Professor Xiaoming Sun and Yale University working with Prof. Hailiang Wang. His research focuses on developing novel transition metal nanostructures for key electrocatalysis processes, such as water splitting and $\mathrm{CO}_{2}$ reduction reactions.
Yaping Li received her BSc degree from the Department of Chemical Engineering and $\mathrm{PhD}$ from the Department of Chemistry, Beijing Institute of Technology in 1996 and 2005, respectively. She joined the State Key Laboratory of Chemical Resource Engineering, Beijing University of Chemical Technology in 2008. Her main research interests focus on the first-principle theory and density functional study on the OER mechanism for metal/metal oxides, and corresponding nonlinear dynamics behaviors.

\section{基于富缺陷镍铁水滑石材料的高效析氧电极}

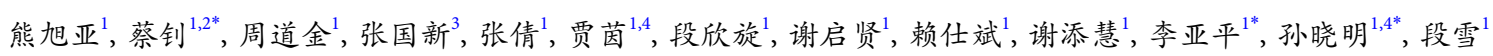

摘要 探索低成本高效率的析氧电极对于工业电解水技术的发展至关重要. 尽管镍铁水滑石已被公认为是一种高效析氧的非贵金属催化 剂, 但其本征活性还有待进一步提高. 本研究通过将氧空位缺陷引入镍铁水滑石, 设计出一种低成本高效率的析氧电极. 通过精确电子结 构调控, 暴露更多活性位点, 提高电极导电性, 富缺陷镍铁水滑石电极展现出 $1.40 \mathrm{~V}$ ( vs. RHE)的低起峰电位. 同时, 它仅需 $200 \mathrm{mV}$ 过电势就 能达到 $10 \mathrm{~mA} \mathrm{~cm}^{-2}$ 的电流密度, 这相比未经处理的镍铁水滑石降低了 $48 \mathrm{mV}$. 我们进一步通过密度泛函理论计算发现, 氧空位缺陷的引入 使Fe的价态降低, 带隙减小, 使得催化过程中电子更容易被激发到导带中, 从而降低反应过电势并使析氧活性增强. 